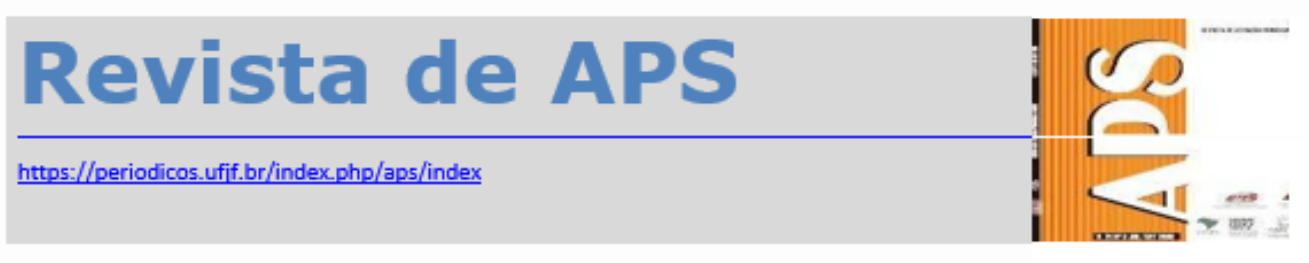

\title{
Adesão ao tratamento medicamentoso da hipertensão arterial em dois modelos de atenção à saúde
}

\author{
Medication adherence to the treatment of hypertension in two \\ models of health care
}

Ana Lúcia de Jesus Almeida ${ }^{1}$, Nathalia Serafim da Silva ${ }^{2}$, Viviane de Freitas Cardoso ${ }^{3}$, Franciele Marques Vanderlei ${ }^{4}$, Renilton José Pizzol ${ }^{5}$, Eliane Ferrari Chagas ${ }^{6}$

\section{RESUMO}

A Unidade Básica de Saúde (UBS) e a Estratégia de Saúde da Família (ESF) são dois modelos diferentes de assistência na Atenção Primária à Saúde. Objetivou-se comparar o grau de adesão a terapêutica medicamentosa de indivíduos com hipertensão assistidos em Estratégia de Saúde da Família (ESF) e Unidade Básica de Saúde (UBS). O estudo teve a participação de 63 indivíduos da ESF e 51 da UBS. Aplicou-se questionários para identificar o perfil da população e avaliar a adesão (teste de Morisky-Green - TMG). Utilizou-se estatística descritiva e, para a análise de associação, os testes Goodman e odds ratio. As variáveis idade, sexo feminino e sedentarismo apresentaram-se homogêneas em ambos os grupos. Entre as variáveis estudadas, apenas a idade mostrou diferença significante, pois pessoas com menos de 60 anos demonstraram menor adesão ao tratamento. Houve diferença significativa na adesão ao tratamento medicamentoso (ESF > UBS). Conclui-se que a melhor adesão foi na ESF, mas ambas foram consideradas abaixo do desejado.

PALAVRAS-CHAVE: Estratégia de Saúde da Família; Unidade Básica de Saúde;

\footnotetext{
${ }^{1}$ Docente no Departamento de Fisioterapia da Faculdade de Ciências e Tecnologia da Universidade Estadual Paulista (Unesp) - campus de Presidente Prudente. E-mail: analu@fct.unesp.br.

${ }^{2}$ Fisioterapeuta. Egressa Universidade Estadual Paulista (Unesp) - campus de Presidente Prudente. Universidade Estadual Paulista (Unesp) - campus de Presidente Prudente.

${ }^{3}$ Enfermeira. Doutoranda no Programa de Pós-Graduação em Fisioterapia na Universidade Federal de São Carlos (UFSCar).

4 Professora Substituta no Departamento de Fisioterapia da Faculdade de Ciências e Tecnologia da Universidade Estadual Paulista (Unesp) - campus de Presidente Prudente.

${ }^{5}$ Docente no Departamento de Fisioterapia da Faculdade de Ciências e Tecnologia da Universidade Estadual Paulista (Unesp) - campus de Presidente Prudente.

${ }^{6}$ Docente no Departamento de Fisioterapia da Faculdade de Ciências e Tecnologia da Universidade Estadual Paulista (Unesp) - campus de Presidente Prudente.
} 
Hipertensão; Adesão ao Tratamento Medicamentoso.

ABSTRACT

The Basic Health Unit (UBS) and the Family Health Strategy (ESF) are two different models of assistance in the Primary Health Care (APS). The objective was to compare therapeutic drug adherence degree in hypertension persons in Family Health Strategy (ESF) and Health Centers (UBS). There were 63 participants from the Family Health Strategy and 51 from the Health Centers. A profile identification questionnaire and a Morisky-Green test (MGT) were used. Descriptive statistic was used and Goodman and Odds Ratio tests were applied for association analyses. Variables as age, female gender and sedentarism were homogeneous in both groups. Among the variables, only age was significantly different, meaning that persons under 60 years of age has have lower rate of adherence to treatment. There was a significative difference in the adhesion (ESF > UBS).

KEYWORDS: Family Health Strategy; Health Centers; Hypertension; Adherence to Medicinal Treatment.

\section{INTRODUÇÃO}

No Sistema Único de Saúde (SUS), a Atenção Primária à Saúde (APS) caracterizase por um conjunto de ações de saúde, no âmbito individual, familiar e da comunidade, abrangendo a prevenção, a promoção e a proteção da saúde, além do diagnóstico, do tratamento e da reabilitação, com o objetivo de desenvolver uma atenção integral que impacte positivamente na situação de saúde das coletividades. ${ }^{1}$ A Unidade Básica de Saúde (UBS) e a Estratégia de Saúde da Família (ESF) compõem a APS, que é a porta de entrada preferencial do SUS. As demandas atendidas nas UBSs apresentam-se como espontâneas e/ou encaminhadas por outros serviços, não havendo, neste caso, adstrição de clientela e delimitação de área de abrangência, ${ }^{2}$ com oferecimento de consultas e serviços de forma programada. ${ }^{3}$ Por outro lado, nas ESFs, a ênfase no território faz parte da proposta de reorientação do modelo assistencial, com o objetivo de desenvolver práticas participativas, planejadas em função da família e não apenas do indivíduo, buscando superar as ações centradas de forma exclusiva nas doenças, a fim de reduzir problemas de saúde através da prevenção e da busca ativa. ${ }^{4}$

Tanto nas UBSs quanto nas ESFs, as doenças crônicas não transmissíveis (DCNT) são prioridades de atendimento e constituem um dos principais problemas de morbidade e mortalidade na sociedade moderna. ${ }^{5}$ Dentro desse contexto, a hipertensão arterial sistêmica (HAS) aparece como sendo a doença mais prevalente, e o tratamento 
não medicamentoso associado ao tratamento medicamentoso tem como objetivo reduzir a pressão arterial para valores inferiores a $140 / 90 \mathrm{mmHg}$, respeitando-se a presença de doenças, condições associadas, características individuais e qualidade de vida do indivíduo. ${ }^{6}$

O Ministério da Saúde, na tentativa de controlar doenças de grande impacto na população, criou programas específicos, entre eles o Programa Nacional de Hipertensão e Diabetes Mellitus (Hiperdia), colocado em prática no ano de $2002 .^{7}$ O programa possibilita aos usuários cadastrados o acompanhamento médico e a garantia do recebimento dos medicamentos prescritos. Assim, evitam-se as complicações decorrentes da não adesão a medicamentos por falta de recursos financeiros, além da adoção de estratégias de saúde pública para a melhoria da qualidade de vida desses indivíduos. $^{8}$

De acordo com Girotto et al. (2013), um dos maiores desafios no combate à HAS deve-se à não adesão ao tratamento medicamentoso. ${ }^{9}$ Sabe-se que o grau de adesão ao medicamento relaciona-se com diferentes fatores, destacando-se o satisfatório nível de informação e o interesse do paciente em comprometer-se no plano terapêutico que exige mudança comportamental e esforço, pois envolve o rompimento com hábitos não saudáveis construídos no decorrer da vida. ${ }^{10}$

Diante desse contexto, vale ressaltar que a concepção de modelo de atenção nas UBSs e ESFs apresentam dinâmicas distintas. ${ }^{1 .}$ Portanto, mais informações sobre aspectos relacionados à adesão ao tratamento medicamentoso são necessárias para possibilitar a identificação da corresponsabilização do sistema de saúde para um tratamento efetivo, no qual a educação em saúde seja acessível e o usuário tenha informações suficientes para aderir ao tratamento proposto pela equipe. Assim, o objetivo do presente estudo foi comparar o grau de adesão terapêutico medicamentoso de indivíduos com HAS assistidos nos dois diferentes modelos de atenção: ESF e UBS.

\section{CASUÍSTICA E MÉTODOS}

O estudo caracteriza-se como transversal e quantitativo. A amostra foi composta por 114 indivíduos com diagnóstico médico de HAS, assistidos em uma ESF ( $n=63$ usuários) e uma UBS ( $n=51$ usuários), localizadas no município de Presidente Prudente, 
em São Paulo.

Foram considerados critérios de inclusão os indivíduos com diagnóstico médico de HAS de ambos os sexos e com idade superior a 18 anos que estavam em tratamento medicamentoso anti-hipertensivo cadastrados no programa Hiperdia da ESF ou indivíduos que buscavam medicamentos para a HAS na UBS. Excluíram-se do estudo os indivíduos que se recusaram a assinar o TCLE (dois indivíduos), com dificuldade na compreensão do questionário (três indivíduos) e os acamados.

Realizou-se a definição dos participantes do estudo na ESF a partir de uma escolha aleatória, utilizando-se da lista de usuários cadastrados como hipertensos. Os dados foram coletados por apenas um pesquisador em visitas domiciliares ou na própria ESF, enquanto aguardavam o atendimento. Já na UBS a abordagem foi realizada no momento em que os usuários iam buscar o medicamento ou enquanto aguardavam consulta médica na sala de espera.

Para a coleta de dados, inicialmente foi elaborado um questionário, com o objetivo de traçar o perfil clínico e sociodemográfico dos participantes. Esse questionário continha informações pessoais, como: sexo, idade, estado civil, escolaridade e frequência de prática de atividade física. Para mensurar o grau de adesão do participante ao tratamento, realizou-se a aplicação de um questionário estruturado denominado teste de Morisky-Green, proposto por Morisky e Green e modificado por Sewicht. ${ }^{11}$

$\mathrm{Na}$ abordagem inicial do participante pelo pesquisador, realizou-se a explicação do estudo. Logo após esse procedimento, os participantes responderam ao questionário do perfil e, na sequência, o teste, também chamado de Teste de Medida de Adesão a Tratamentos (MAT). A duração do preenchimento completo dos questionários foi de, aproximadamente, 10 minutos. Vale destacar que as entrevistas foram realizadas de forma individual, reservadamente, e conduzidas pelo pesquisador.

O MAT modificado é um questionário fundamentado em quatro itens. A pontuação obtida varia de 0 a 4, atribuindo-se o valor 1 a cada resposta negativa. Consideram-se alta adesão ao tratamento o escore de 4 pontos; média adesão, os escores de 2 a 3 pontos; e baixa adesão, os escores de 0 a 1 ponto. As quatro perguntas iniciais do MAT modificado permitem classificar em dois tipos o comportamento do indivíduo com predisposição para não adesão. Os que respondem "sim" às questões 1 
e/ou 2 caracterizam-se como tendo comportamento de não adesão do tipo não intencional e aqueles que respondem "sim" às questões 3 e/ou 4 são classificados como tendo comportamento do tipo intencional. ${ }^{11}$

Aos participantes que respondem "sim" a pelo menos uma dessas questões iniciais, realizam-se duas perguntas adicionais com o objetivo de determinar o motivo associado ao comportamento de não adesão. Os que responderam "não" à questão 5 são considerados participantes que não têm conhecimento sobre a importância do tratamento. Se a resposta for "sim" na questão 6, a falta de motivação é considerada motivo da não adesão ao tratamento. ${ }^{11}$

Para a análise dos dados do perfil da população e a descrição das variáveis, utilizou-se o método estatístico descritivo. Os resultados foram apresentados com valores de média, desvio-padrão, intervalo de confiança, percentuais e números absolutos.

A investigação da associação entre o perfil da população e a adesão ao medicamento de acordo com os modelos de atenção ao indivíduo (ESF e UBS) foi realizada por meio do teste de Goodman para contrastes entre e dentro de populações binomiais e/ou multinomiais, conforme a característica do grupo de variáveis a ser analisada. Utilizaram-se também o odds ratio e o intervalo de confiança de 95\% (IC95\%) para detectar se existia a relação de determinadas variáveis na adesão ou na não adesão ao medicamento. Considerou-se nível de 5\% de significância para todas as análises.

O estudo foi aprovado pelo Comitê de Ética local (CAAE: 41034914.1.0000.5402), atendendo aos requisitos da Resolução 466/12 e às suas complementares do Conselho Nacional de Saúde. Os participantes do estudo realizaram a leitura, a compreensão e a autorização por escrito no Termo de Consentimento Livre e Esclarecido.

\section{RESULTADOS}

A população assistida na ESF apresentou média de idade de 69,16 $\pm 9,26$ anos e, na UBS, 63,25 $\pm 8,64$ anos. Isso apontou uma população mais jovem na UBS. A Tabela 1 mostra os dados obtidos das variáveis pessoais, de acordo com a unidade de atendimento. Em relação ao sexo, a maioria dos entrevistados foi do sexo feminino em ambas as unidades. Além disso, houve diferença significante no número de mulheres 
em relação aos homens em ambas as unidades. Na comparação entre as unidades, 0 sexo feminino é mais frequente na ESF (odds ratio: 0,20; IC95\%: 0,12-0,66).

Tabela 1 - Frequência absoluta (n) e relativa (\%) por sexo, faixa etária, estado civil, escolaridade e da prática de atividade física de acordo com a unidade de atendimento

\begin{tabular}{|c|c|c|c|}
\hline Variáveis & $\operatorname{ESF}(n=63)$ & UBS $(n=51)$ & Total $(n=114)$ \\
\hline \multicolumn{4}{|l|}{ Sexo } \\
\hline Masculino & $12(19,05) *$ & $23(45,10)$ & $35(30,70)$ \\
\hline Feminino & $51(80,95) * \#$ & $28(54,90)$ & $79(69,30)$ \\
\hline Total & $63(100,0)$ & $51(100,0)$ & $114(100,0)$ \\
\hline \multicolumn{4}{|l|}{ Faixa etária } \\
\hline$\leq 59$ anos & $9(14,29) * \Delta$ & $19(37,26)$ & $28(24,56)$ \\
\hline $60-69$ anos & $21(33,33)$ & $16(31,37)$ & $37(32,46)$ \\
\hline$\geq 70$ anos & $33(52,38)$ & $16(31,37)$ & $49(42,98)$ \\
\hline Total & $63(100,0)$ & $51(100,0)$ & $114(100,0)$ \\
\hline \multicolumn{4}{|l|}{ Estado civil } \\
\hline Solteiro & $3(4,76)$ & $4(7,84)$ & $7(6,15)$ \\
\hline Casado & $36(57,15) ¥$ & $34(66,67) \#$ & $70(61,40)$ \\
\hline Separado & $3(4,76)$ & $6(11,76)$ & $9(7,89)$ \\
\hline Viúvo & $21(33,33) * ¥$ & $7(13,73)$ & $28(24,56)$ \\
\hline Total & $63(100,0)$ & $51(100,0)$ & $114(100,0)$ \\
\hline \multicolumn{4}{|l|}{ Anos de estudo } \\
\hline$<1$ ano & $13(20,64)$ & $6(11,77)$ & $19(16,67)$ \\
\hline 1 a 5 anos & $36(57,14) \#$ & $27(52,94)+$ & $63(55,26)$ \\
\hline 6 a 10 anos & $7(11,11)$ & $5(9,80)$ & $12(10,53)$ \\
\hline$>10$ anos & $7(11,11)$ & $13(25,49)$ & $20(17,54)$ \\
\hline Total & $63(100,0)$ & $51(100,0)$ & $114(100,0)$ \\
\hline \multicolumn{4}{|c|}{ Frequência da prática de atividade física } \\
\hline $0-1 \mathrm{x} /$ semana & $40(63,49) \#$ & $28(54,90) \infty$ & $68(59,65)$ \\
\hline $2-4 x /$ semana & $13(20,64)$ & $14(27,45)$ & $27(23,68)$ \\
\hline $5-7 x /$ semana & $10(15,87)$ & $9(17,65)$ & $19(16,67)$ \\
\hline Total & $63(100,0)$ & $51(100,0)$ & $114(100,0)$ \\
\hline \multicolumn{4}{|c|}{$\begin{array}{l}\text { *: Diferença estatisticamente significante em relação à UBS; \#: Diferença estatisticamente significante } \\
\text { em relação às demais categorias da variável para o mesmo grupo (ESF/UBS); } \Delta \text { : Diferença } \\
\text { estatisticamente significante em relação à faixa etária acima de } 70 \text { anos; ¥: Diferença estatisticamente } \\
\text { significante em relação ao solteiro e separado; †: Diferença estatisticamente significante em relação à } \\
\text { escolaridade < } 1 \text { ano e } 6 \text { a } 10 \text { anos; } \infty \text { : Diferença estatisticamente significante em relação à frequência } \\
\text { de } 5-7 \text { vezes/semana }\end{array}$} \\
\hline
\end{tabular}

Fonte: elaborada pelos autores

Quanto à escolaridade, a ESF apresentou maior número de pessoas com até cinco anos de estudo, enquanto na UBS a escolaridade foi maior, porém a amostra não difere entre ambas. Em relação ao estado civil, nas duas unidades os entrevistados eram, em sua maioria, casados. Na ESF, houve um destaque para o percentual de viúvos. Em relação à frequência da prática de atividade física, observou-se que a população 
estudada é sedentária em ambas as unidades. Todas essas características demonstram que, apesar de a amostra ter sido realizada de forma aleatória, há poucas diferenças estatisticamente significativas nas variáveis que caracterizaram o perfil populacional.

Os dados do questionário de adesão MAT estão apresentados na Tabela 2 com a análise sobre o resultado dicotômico de adesão e não adesão e a classificação por escore. Houve diferença significativa na variável adesão ao tratamento em relação à ESF e à UBS, apontando maior adesão na ESF (odds ratio: 2,40; IC95\%: 1,09 - 5,29). No entanto, a não aderência ao tratamento é significativa, seja na ESF seja na UBS. Na ESF, observou-se que não houve diferença estatisticamente significante entre os usuários que aderem ou não ao tratamento, enquanto, na UBS, o número de participantes que não aderem é significativamente maior. Na ESF, dos que aderem, há média e alta capacidades de adesão. Essa classificação prevalece como média adesão na UBS.

Tabela 2 - Frequência absoluta ( $\mathrm{n}$ ) e relativa (\%) dos dados do MAT dos indivíduos com diagnóstico médico de hipertensão arterial, de acordo com a unidade de atendimento

\begin{tabular}{|c|c|c|c|}
\hline Variáveis & $\operatorname{ESF}(n=63)$ & UBS $(n=51)$ & Total $(n=114)$ \\
\hline \multicolumn{4}{|l|}{ MAT } \\
\hline Adere & $30(47,62) *$ & $14(27,45)$ & $44(38,60)$ \\
\hline Não adere & $33(52,38) *$ & $37(72,55) \#$ & $70(61,40)$ \\
\hline Total & $63(100,0)$ & $51(100,0)$ & $114(100,0)$ \\
\hline \multicolumn{4}{|c|}{ Classificação da adesão (escore) } \\
\hline Baixa & $5(7,94) \#$ & $4(7,85)$ & $9(7,89)$ \\
\hline Média & $28(44,44)$ & $33(64,70) \#$ & $61(53,51)$ \\
\hline Alta & $30(47,62)$ & $14(27,45)$ & $44(38,60)$ \\
\hline Total & $63(100,0)$ & $51(100,0)$ & $114(100,0)$ \\
\hline
\end{tabular}

Fonte: elaborada pelos autores

Na Tabela 3, analisou-se o comportamento do participante com predisposição para a não adesão (intencional ou não intencional). Não foi observada diferença entre essas classificações (odds ratio: 0,82; IC95\%: 0,31 - 2,19). O motivo da não adesão não se relacionou neste estudo com falta de motivação ou conhecimento. Os participantes de ambas as unidades apresentaram-se, em sua maioria motivados, e diziam conhecer a importância de tomar o medicamento. Apesar disso, vale destacar que, na UBS (78,38\%), esse motivo da não adesão mostrou-se menor que na $\operatorname{ESF}(81,82 \%)$. 
Tabela 3 - Frequência absoluta (n) e relativa (\%) dos tipos de não adesão ao tratamento, segundo MAT, de acordo com a unidade de atendimento

\begin{tabular}{llll}
\hline Variáveis & ESF $(\mathbf{n}=\mathbf{3 3})$ & UBS $(\mathbf{n}=\mathbf{3 7})$ & Total \\
\hline Não adesão & & & \\
Tipo intencional & $11(33,33)$ & $14(37,84)$ & $25(35,71)$ \\
Tipo não intencional & $22(66,67)$ & $23(62,16)$ & $45(64,29)$ \\
Total & $33(100,0)$ & $37(100,0)$ & $70(100,0)$ \\
Motivo da não adesão & & & \\
Falta apenas de conhecimento & $3(9,09)$ & $4(10,81)$ & $7(10,00)$ \\
Falta apenas de motivação & $3(9,09)$ & $3(8,11)$ & $6(8,57)$ \\
Falta de conhecimento e motivação & $0 *$ & $1(2,70)$ & $1(1,43)$ \\
Tem conhecimento e motivação & $27(81,82) \#$ & $29(78,38) \#$ & $56(80)$ \\
Total & $33(100,0)$ & $37(100,0)$ & $70(100,0)$ \\
\hline \hline *; Diferença estatisticamente significante em relação à UBS; \#: Diferença estatisticamente significante \\
em relação às demais categorias da variável na mesma unidade de atendimento.
\end{tabular}

Fonte: elaborada pelos autores

A Tabela 4 mostra os resultados da adesão ao tratamento distribuída de acordo com as características do perfil. Para as variáveis sexo (odds ratio: 0,64; IC95\%: 0,28 1,48), estado civil, escolaridade e frequência da prática de atividade física, não foram observadas diferenças estatisticamente significantes que pudessem influenciar na adesão ao tratamento. Apenas em relação à idade houve diferença estatística na não adesão para participantes com menos de 60 anos.

Tabela 4 - Frequência absoluta ( $\mathrm{n}$ ) e relativa (\%) da adesão ao tratamento de acordo com o sexo, faixa etária, estado civil, anos de estudo e frequência da prática de atividade física na população estudada

\begin{tabular}{|c|c|c|c|}
\hline Variáveis & $\begin{array}{l}\text { Adere } \\
44(38,6 \%)\end{array}$ & $\begin{array}{l}\text { Não adere } \\
70(61,4 \%)\end{array}$ & $\begin{array}{l}\text { Total } \\
114(100,0 \%)\end{array}$ \\
\hline \multicolumn{4}{|l|}{ Sexo } \\
\hline Masculino & $11(25,00)$ & $24 \#(34,28)$ & $35(30,70)$ \\
\hline Feminino & $33(75,00)$ & $46(65,71)$ & $79(69,30)$ \\
\hline \multicolumn{4}{|l|}{ Faixa etária } \\
\hline$\leq 59$ anos & $5(11,36) * \#$ & $23(32,86)$ & $28(24,56)$ \\
\hline $60-69$ anos & $18(40,91)$ & $19(27,14)$ & $37(32,46)$ \\
\hline$\geq 70$ anos & $21(47,73)$ & $28(40,00)$ & $49(42,98)$ \\
\hline \multicolumn{4}{|l|}{ Estado Civil } \\
\hline Solteiro & $1(2,27)$ & $6(8,57)$ & $7(6,14)$ \\
\hline Casado & $27(61,36)$ & $43(61,43) \#$ & $70(61,40)$ \\
\hline Separado & $4(9,10)$ & $5(7,14)$ & $9(7,90)$ \\
\hline Viúvo & $12(27,27)$ & $16(22,86)$ & $28(24,56)$ \\
\hline
\end{tabular}


(Conclusão)

\begin{tabular}{|c|c|c|c|}
\hline Variáveis & $\begin{array}{l}\text { Adere } \\
44(38,6 \%)\end{array}$ & $\begin{array}{l}\text { Não adere } \\
70(61,4 \%)\end{array}$ & $\begin{array}{l}\text { Total } \\
114(100,0 \%) \\
\end{array}$ \\
\hline \multicolumn{4}{|l|}{ Anos de Estudo } \\
\hline$<1$ ano & $8(18,18)$ & $11(15,72)$ & $19(16,67)$ \\
\hline 1 a 5 anos & $24(54,55)+$ & $39(55,72) \#$ & $63(55,26)$ \\
\hline 6 a 10 anos & $3(6,82)$ & $9(12,86)$ & $12(10,53)$ \\
\hline$>10$ anos & $9(20,45)$ & $11(15,71)$ & $20(17,54)$ \\
\hline \multicolumn{4}{|c|}{ Frequência da Prática de Atividade Física } \\
\hline $0-1 \times /$ semana & $25(56,81)$ & $43(61,42)$ & $68(59,65)$ \\
\hline $2-4 x /$ semana & $11(25,00)$ & $16(22,86)$ & $27(23,68)$ \\
\hline $5-7 x /$ semana & $8(18,18)$ & $11(15,71)$ & $19(16,66)$ \\
\hline
\end{tabular}

*: Diferença estatisticamente significante em relação aos indivíduos que não aderem ao tratamento; \#: Diferença estatisticamente significante em relação às demais categorias da variável para o mesmo grupo (adere/não adere); $\mathbf{C}$ : Diferença estatisticamente significante em relação aos estados civis casado e viúvo; : Diferença estatisticamente significante em relação ao estado civil casado; †: Diferença estatisticamente significante em relação à escolaridade $<1$ ano e 6 a 10 anos

Fonte: elaborada pelos autores

\section{DISCUSSÃO}

O presente estudo teve por finalidade comparar o grau de adesão terapêutica medicamentosa de indivíduos com HAS entre a ESF e a UBS. Os resultados obtidos mostraram que a adesão é maior na ESF do que na UBS e que, em ambos os modelos, ela se mostrou preocupante (47,6\% de indivíduos na ESF e $27,4 \%$ na UBS). Tal fato corrobora a observação de que a adesão aos medicamentos em indivíduos com doenças crônicas é baixa, devido a vários fatores, desde os individuais (idade, situação econômica) até aos relacionados ao próprio tratamento e ao acesso ao sistema de saúde. ${ }^{12}$ Outro estudo observou que a adesão ao tratamento foi reduzida em um grupo de idosos acompanhados no atendimento primário e um dos fatores seria a falta de simplificação de alguns esquemas terapêuticos. ${ }^{13}$

A diferença de adesão entre a ESF e a UBS observada neste estudo indica que o nível de adesão aos medicamentos pode ter sofrido influência direta do modelo de atenção, conforme já preconizado na literatura. ${ }^{14}$ Assim, pode-se considerar que o modelo de atenção à saúde disponibilizado na ESF justificaria o maior nível de adesão, contrapondo-se ao nível de adesão na UBS.

Este resultado pode estar associado ao fato de que, dentro da APS, a ESF pode ser considerada o espaço prioritário de garantia de acompanhamento mais sistemático 
de indivíduos com HAS com a elaboração de ações de promoção de saúde como o Programa Hiperdia, que garante a necessidade de um acompanhamento mensal para os usuários, para educação em saúde e orientação. Desse modo, conscientiza-os sobre as mudanças no estilo de vida e a importância na adesão aos tratamentos propostos, para melhorar a qualidade de vida e evitar o aparecimento de complicações. ${ }^{15}$

De acordo com os resultados do estudo realizado por Remondi et al. (2014), quanto às variáveis associadas a não adesão, foram apontadas três: não ser acompanhado pelos agentes comunitários de saúde (ACS), ter tido descontinuidade no acesso aos medicamentos e a elevada complexidade do número de doses diárias. Isso reforça a necessidade do acompanhamento contínuo em benefício da adesão, como ocorre na ESF. ${ }^{16}$ No caso do ACS, sua importância reside no fato de que esse profissional estabelece o vínculo entre a população e os profissionais da saúde no território, ${ }^{16}$ o que pode facilitar o acompanhamento terapêutico. Outros estudos identificaram uma associação entre a adesão e o papel do médico e a quantidade de medicamentos utilizados, ${ }^{17}$ reforçando a importância da interação profissional/paciente.

Outros estudos apontam que as características inerentes à ESF, como a boa relação entre os profissionais de saúde e o vínculo destes profissionais com a população assistida, se mostram importantes para estimular o indivíduo a cuidar de sua saúde, promovendo uma melhor adesão ao tratamento, não só dos indivíduos hipertensos, mas de toda a comunidade, ${ }^{18,19}$

Este estudo investigou a associação da adesão a variáveis individuais e sociodemográficas conhecidas por influenciarem a adesão. Com relação ao sexo, observou que os homens apresentaram uma tendência maior à não adesão que as mulheres. Saccomann et al. (2015), ao analisarem os fatores associados à adesão ao tratamento medicamentoso, observaram que os homens tendem a ser mais descuidados em relação à tomada dos medicamentos que as mulheres, o que pode ser justificado pela menor preocupação com a saúde. ${ }^{20}$ Outros estudos apontam que os homens, em sua maioria, não costumam procurar o serviço de saúde, potencializando a subestimação de suas doenças e seus agravos e dificultando a adesão terapêutica. ${ }^{21,22}$

Neste estudo, os usuários da UBS eram mais jovens, com maior escolaridade e aderiram menos ao tratamento medicamentoso, mantendo-se entre baixa e média adesão $(72,5 \%)$, enquanto na ESF os usuários apresentaram-se entre média e alta 
adesão (92\%). Em relação à idade, existem controvérsias na literatura quanto à influência da faixa etária na adesão. Enquanto alguns estudos apontam uma ausência de relação entre a faixa etária, ${ }^{17}$ outros indicam maior adesão ao tratamento de indivíduos com mais idade. ${ }^{8,23}$ Essa controvérsia pode estar relacionada a fatores que favorecem a adesão como a preocupação do idoso hipertenso com as comorbidades decorrentes e com o aparecimento de outras complicações e a fatores que a dificultam como características inerentes à idade (déficit de memória; perdas da função visual e cognitiva da acuidade visual que interferem com a manipulação do medicamento) e esquema terapêutico (número excessivo de medicamentos por causa de outras condições crônicas e seus efeitos colaterais). ${ }^{13,22,24,25}$ Outro estudo que apontou que indivíduos de 20 a 59 anos apresentaram risco 2,5 vezes maior à não adesão medicamentosa, quando comparados aos indivíduos com 60 anos ou mais, concluiu que a não adesão estava relacionada à característica assintomática da doença. ${ }^{26}$

Em relação aos anos de estudo, a literatura aponta que a baixa escolaridade influencia negativamente na adesão ao tratamento, seja pela falta de conhecimento seja por piores condições financeiras. ${ }^{27}$ Neste estudo, os indivíduos com escolaridade entre 1 a 5 anos estavam entre os que mais não aderiram ao tratamento. Provavelmente, tal fato ocorreu porque a opção de seleção da amostra foi aleatória, e a maioria dos indivíduos entrevistados estava nessa faixa de escolaridade. Isso indica que os indivíduos entrevistados tinham baixa escolaridade que poderia ter influenciado na baixa adesão observada tanto na ESF quanto na UBS.

A adesão à atividade física foi baixa em ambas as unidades. A tendência de pacientes que realizavam atividades físicas apresentarem adesão total ao tratamento farmacológico apontada no estudo de Aragão et al. (2011) vai ao encontro dos resultados desse estudo. ${ }^{28}$

No presente estudo, a não adesão do tipo não intencional foi a mais prevalente nas duas unidades, classificada pelo descuido com o horário e/ou esquecimento. Em estudos que investigaram os tipos de não adesão, os principais motivos alegados pelos indivíduos que não aderiram ao tratamento foi o esquecimento, a convicção de que a pressão arterial estava controlada e o atraso no uso do medicamento. ${ }^{8,23}$ Tal fato revelando a necessidade de estabelecer medidas que permitam ao hipertenso compreender adequadamente sua doença e a importância de mudanças 
comportamentais em seu dia a dia. ${ }^{8,23}$

Neste estudo, apesar do tipo não intencional ter sido o mais prevalente nas duas unidades, a falta de conhecimento e a de motivação não foram razões da não adesão. Assim, fica evidente que a não adesão está associada a motivos não intencionais.

Esses resultados servem como indicadores para o planejamento de ações estratégicas, principalmente na UBS, destacando-se a necessidade de fortalecer a implementação de ações que incluam a discrição de clientela, visando ao estabelecimento de um vínculo com essa população. A prática de modelos assistenciais mais coerentes com os princípios da universalidade, da equidade e da integralidade da atenção deve permear o trabalho na ABS. Desse modo, contribui-se para a adesão ao tratamento, proporcionando ferramentas para o combate a não adesão, além da implementação de programas multidisciplinares em todos os níveis de atendimento aos pacientes hipertensos, que visem à educação da população para as intervenções serem mais eficazes. Isso reduziria a prevalência de não adesão, proporcionando melhor qualidade de vida e redução de eventos cardiovasculares futuros. ${ }^{17}$

\section{CONCLUSÃO}

Por fim, houve maior adesão ao tratamento medicamentoso dos indivíduos hipertensos assistidos na ESF em comparação com os indivíduos assistidos na UBS. Contudo, nas duas unidades, a adesão ao tratamento foi considerada abaixo do desejado. Além disso, verificou-se que a motivação e o conhecimento não foram razões para a não adesão e que apenas a idade mostrou diferença estatística na não adesão para participantes com menos de 60 anos.

Essas características reforçam a hipótese de que o modelo de atenção na ESF deve ser o principal fator de influência na maior adesão identificada neste estudo. Dessa maneira, ressaltando que, na APS, a educação em saúde torna-se imprescindível, pois não é possível o controle adequado da pressão arterial se o usuário não for instruído sobre os princípios em que são fundamentados o tratamento. Portanto, um modelo mais próximo da população atendida, que tenha a busca ativa como princípio, parece ter uma relação com a maior adesão ao tratamento na hipertensão arterial.

Como limitações do estudo, pode-se citar a falta de randomização da amostra. 
Além disso, não há um consenso sobre um método para avaliar a adesão que possa ser tomado como padrão-ouro, e o instrumento utilizado tem suas limitações, pois, apesar de apresentar desempenho variável, pode levar a imprecisões na discriminação da adesão/não adesão.

\section{REFERÊNCIAS}

1. Brasil. Secretaria de Atenção à Saúde. Departamento de Atenção Básica. Política Nacional de Atenção Básica [Internet]. Brasília: Departamento de Atenção Básica; 2012. [citado 2019 nov 30]. Disponível em: http://dab/docs/publicacoes/geral/pnab.pdf.

2. Elias PE, Ferreira CW, Alves MCG, Cohn A, Kishima V, Júnior AE et al. Atenção Básica em Saúde: Comparação entre PSF e UBS por estrato de exclusão social no município de São Paulo. Ciênc Saúde Colet. 2006; 11 (3): 633-641. Disponível em: http://www.scielo.br/scielo.php?pid=S141381232006000300012\&script=sci_abstract \&tlng=pt.

3. Melo DO, Silva SRA, Castro LLC. Avaliação de indicadores de qualidade de prescrição de medicamentos em uma unidade de atenção primária com diferentes modelos de atenção. Epidemiol Serv Saúde. 2016; 25 (2): 259-270.

4. Araújo MCF, Alves MS, Padre PMM, Andrade DM, Silva ACM, Barreto LP et al. Perfil de não adesão ao tratamento de usuários com diabetes e hipertensão em uma Unidade de Saúde da Família. Ciênc Biol Agrar Saúde. 2016; 20(1):43-48.

5. Lessa, I. Doenças crônicas não transmissíveis. In: Berlezi EM, Franz LB (ed.). Doenças e agravos não transmissíveis. ljuí: Unijiuí; 2011. p. 15-45.

6. Sociedade Brasileira de Hipertensão. VI Diretrizes Brasileiras de Hipertensão Arterial. Arq Bras Cardiol. 2010 ; 95 (1): 1-51.

7. Carvalho ALM, Leopoldino RWD, Silva JEG, Cunha CP. Adesão ao tratamento medicamentoso em usuários cadastrados no Programa Hiperdia no município de Teresina (PI). Ciên Saúde Colet, 2012; 17 (7):1885-1892.

8. Reis AFN, Cesarino CB. Fatores de risco e complicações em usuários cadastrados no Hiperdia de São José do Rio Preto. Ciênc Cuid Saúde. 2016; 15 (1):118-124.

9. Girotto E, Andrade SM, Cabrera MAS, Matsuo T. Adesão ao tratamento farmacológico 
e não farmacológico e fatores associados na atenção primária da hipertensão arterial. Ciên Saúde Colet. 2013; 18 (6): 1763-1772.

10. Marin NS, Santos MF, Moro AS. Percepção de hipertensos sobre a sua não adesão ao uso de medicamentos. Rev Esc Enferm USP. 2016; 50 (esp): 061-067.

11. Cornélio RCAC, Pinto ALT, Pace FHL, Moraes JP, Chebli JMF. Nãoadesão ao tratamento em pacientes com doença de Crohn: prevalência e fatores de risco. Arq. Gastroenterol. 2009 ; 46 (3): 183-189.

12. Mansour SN, Monteiro CN, Luiz ODC. Adesão ao tratamento farmacológico de pacientes hipertensos entre participantes do Programa Remédio em Casa. Epidemiol Serv Saúde. 2016 ; 25 (3): 647-654.

13. Bastos-Barbosa RG, Ferrioli E, Moriguti JC, Nogueira CB, Nobre F, Ueta J et al. Adesão ao tratamento e controle da pressão arterial em idosos com hipertensão. Arq Bras Cardiol. 2012; 99 (1): 636-641.

14. Dias T K, Netto ACMG, Teixeira AL, Soares G, Almeida FP, Costa JC et al. Adesão ao tratamento medicamentoso da hipertensão em um programa de saúde da família. Geriatr Gerontol Aging. 2014; 8 (2): 110-115.

15. Lima AS, Gaia ESM, Ferreira MA. A importância do Programa Hiperdia em uma Unidade de Saúde da Família do município de Serra Talhada - PE, para adesão dos hipertensos e diabéticos ao tratamento medicamentoso e dietético. Saúde Coletiva Deb. 2012 ; 2(1): 09-17.

16. Remondi FA, Cabrera MAS, Souza RKT. Não adesão ao tratamento medicamentoso contínuo: prevalência e determinantes em adultos de 40 anos e mais. Cad Saúde Públ. 2014; 30 (1): 126-136.

17. Eid LP, Nogueira MS, Veiga EV, Cesarino EJ, Alves LMM. Adesão ao tratamento antihipertensivo: análise pelo Teste de Morisky-Green. Rev Eletr Enf. 2013 ; 15 (2): 362367. [citado 2019 nov 30]. Disponível em: http://dx.doi.org/10.5216/ree.v15i2.15599.

18. Oshiro ML, Castro L LC, Cymrot R. Fatores para não adesão ao programa de controle da hipertensão arterial em Campo Grande, MS. Rev Cienc Farm Básica Apl. 2010; 31 (1): 95-100.

19. Viegas SMF, Penna CMM. A construção da integralidade no trabalho cotidiano da equipe saúde da família. Esc Anna Nery. 2013 ; 17(1): 133-141.

20. Saccomann ICR, Neta JGS, Martins BF. Fatores associados à adesão ao tratamento 
medicamentoso em hipertensos de uma unidade de saúde da família. Rev Fac Cienc Med Sorocaba. 2015; 17(1): 21-26.

21. Ribeiro IJS, Boery RNSO, Casotti CA, Freire IV, Teixeira JRB, Boery EM. Prevalência e fatores associados à adesão ao tratamento medicamentoso por pacientes com hipertensão arterial. Rev Baiana Enferm. 2015 ; 29 (3): 250-260.

22. Siqueira BPJ, Teixeira JRB, Neto PFV, Boery EN, Boery RNSO, Vilela ABA. Men and health care in the social representations of health professionals. Esc Anna Nery. 2014; 18 (4): 690-696.

23. Tavares DMS, Guimarães MO, Ferreira PCS, Dias FA, Martins NPF, Rodrigues LR. Qualidade de vida e adesão ao tratamento farmacológico entre idosos hipertensos. Rev Bras Enferm. 2016 ; 69 (1): 134-141. [citado 2019 nov 30]. Disponível em: http://www.scielo.br/scielo.php?script=sci_arttext\&pid=S0034$71672016000100134 \&$ Ing=en.

24. Gomes e Martins A, Chavaglia SRR, OhI RIB, Martins IML, Gamba MA. Adesão ao tratamento clínico ambulatorial da hipertensão arterial sistêmica. Acta Paul Enferm. 2014 ; 27(3):266-272. Disponível em: http://www.scielo.br/scielo.php?script=sci_arttext\&pid=S010321002014000300266\&lng=en.

25. Oliveira TS, Oliveira FM, Almeida F, Jandotti AC, Yamaguchi MU, Ramos ERP. Preditores do grau de conhecimento sobre hipertensão arterial em pacientes atendidos pelo Hiperdia de Lagarto- SE. Rev IPI. 2016; 2 (1): 1-15.

26. Magnabosco P, Teraoka EC, Oliveira EM, Felipe EA, Freitas D, Marchi-Alves LM. Análise comparativa da não adesão ao tratamento medicamentoso da hipertensão arterial sistêmica em população urbana e rural. Rev Latino-Am Enferm. 2015; 23(1): 20-27.

27. Silva FO, Suto CSS, Costa LEL. Perfil de pacientes cadastrados no Hiperdia: Conhecendo o estilo de vida. Rev Saúde Col UEFS. 2015; 5 (1): 33-39.

28. Aragão CCV, Santos BRM, Sousa DA, Diniz NB, Garcia MLB, Braga ALF et al. Adesão ao tratamento farmacológico, controle da pressão arterial e qualidade de vida em pacientes hipertensos. In: Encontro de Iniciação Científica da USCS, 1., 2011, São Caetano do Sul. Anais... São Caetano do Sul, SP: Universidade Municipal de São Caetano do Sul, 2011,. [citado 2019 nov 30]. Disponível em: http://www.uscs.edu.br/pesquisasacademicas/images/download_inici_cientifica/prof _brigitte_rieckmann_e_daniela_araujo.pdf. 
Submissão: junho de 2017.

Aprovação: dezembro de 2018. 